\title{
The effect of the humanistic care teaching model on nurse patient conflict and nurse turnover intention in a pediatric outpatient department: results of a randomized trial
}

\author{
Xiaoli Zhong', Xiuzhi Liu' ${ }^{2}$ Yan Sheng ${ }^{3}$ \\ ${ }^{1}$ Department of Child Healthcare, Xiangyang No. 1 People's Hospital, Hubei University of Medicine, Xiangyang, China; ${ }^{2}$ Vaccination Clinic, \\ Juxian Hospital of Traditional Chinese Medicine, Rizhao, China; ${ }^{3}$ Department of Respiratory and Critical Care Medicine, Xiangyang No. 1 People's \\ Hospital, Hubei University of Medicine, Xiangyang, China \\ Contributions: (I) Conception and design: X Zhong; (II) Administrative support: Y Sheng; (III) Provision of study materials or patients: X Lin, \\ Y Sheng; (IV) Collection and assembly of data: Y Sheng; (V) Data analysis and interpretation: Y Sheng; (VI) Manuscript writing: All authors; \\ (VII) Final approval of manuscript: All authors. \\ Correspondence to: Yan Sheng. Department of Respiratory and Critical Care Medicine, Xiangyang No. 1 People's Hospital, Hubei University of \\ Medicine, No. 15 Jiefang Road, Xiangyang 441000, China. Email: nin3400@163.com.
}

Background: This study was carried out based on the background that sharp nurse-patient conflicts in the pediatric outpatient department lead to a high turnover rate of nurses.

Methods: A total of 68 nurses working in the pediatric outpatient department of Xiangyang No. 1 People's Hospital were selected as the study subjects, and randomly divided into an experimental group ( $\mathrm{n}=34$ ) and a control group ( $\mathrm{n}=34)$. Nurses in the control group received a traditional pediatric nursing teaching model, while those in the experimental group received a traditional pediatric nursing teaching model combined with the humanistic care teaching model. The effect of these two nursing teaching models on nurse-patient conflicts in the pediatric outpatient department and the turnover intention of nurses was then compared and analyzed.

Results: There were no significant differences in personal information between the two groups $(\mathrm{P}>0.05)$. The strain capacity, operational capacity, nurse-patient communication skills, autonomous learning ability, and teamwork ability of the nurses in the experimental group after training were significantly higher than those in the control group $(\mathrm{P}<0.05)$. Both groups after training had significantly higher scores on a professional identity scale than before training, and nurses in the experimental group had significantly higher scores of professional identity than those in the control group $(\mathrm{P}<0.001)$. The turnover intention of the nurses in the experimental group were significantly lower than those in the control group $(\mathrm{P}<0.001)$. The problem solving ability of nurses in the experimental group was significantly better than that in the control group $(\mathrm{P}<0.001)$. Scores in the domains of waiting to see the doctor, the health knowledge education, the ward environment, and nursing quality of nurses in 'the experimental group were significantly higher than those in the control group $(\mathrm{P}<0.001)$.

Conclusions: The humanistic care teaching model can significantly improve the professional identity and problem solving ability of nurses in facing different nurse-patient conflicts with significant effect and is worthy of application and popularization in clinical nursing teaching.

Trial Registration: Chinese Clinical Trial Registry ChiCTR2100048751.

Keywords: Humanistic care teaching model; pediatric outpatient department; nurse-patient conflict; turnover intention; effect

Submitted Mar 16, 2021. Accepted for publication Aug 01, 2021.

doi: $10.21037 / \mathrm{tp}-21-214$

View this article at: https://dx.doi.org/10.21037/tp-21-214 


\section{Introduction}

The pediatric outpatient department is a complex and stressful environment. The sense of helplessness and uncertainty felt by family members combined with the demands on staff can easily stretch the emotions of all and lead to nurse-patient/family member/management conflicts which can indirectly lead to a high turnover rate of nurses in the workplace $(1,2)$. At present, the loss of nursing talent has increased the cost of personnel training in Xiangyang No. 1 People's Hospital, resulting in a decline in the level of clinical nursing care. Although our country has introduced a variety of laws, regulations, and policies to protect medical staff, it is still difficult to maintain a stable health care workforce. A shortage of nursing staff is still the biggest problem in the development of Xiangyang No. 1 People's Hospital management and nursing quality, and how to reduce nurse-patient conflicts and the turnover rate of nurses requires urgent attention (3-5). Pediatric patients may be unable to communicate details of their conditions for a variety of reasons, which may result in poor reliability of the information of their health history and more difficulties in nursing assessment, further increasing demands on hospital nursing care $(6,7)$. The humanistic care teaching model is patientcentered and can greatly satisfy the psychological and physical demands of patients during hospitalization, with obvious treatment efficacy, effectively improving nursing comfort, reducing the incidence of clinical adverse events, minimizing the occurrence of nurse-patient conflicts, and promoting the nursing care to a higher level (8). We sought to further analyze the effect of the humanistic care teaching model on nurse-patient conflicts in the pediatric outpatient department and the turnover intention of nurses at Xiangyang No. 1 People's Hospital.

We present the study in accordance with the CONSORT reporting checklist (available at https://dx.doi.org/10.21037/ tp-21-214).

\section{Methods}

\section{General information}

We selected 68 nurses working in the pediatric outpatient department of Xiangyang No.1 People's Hospital from September 2018 to September 2019 and randomly divided them into an experimental group $(n=34)$ and control group $(\mathrm{n}=34)$. This study is two-parallel assay.

\section{Inclusion criteria}

(I) Nurses had the Practicing Nurse Certificate of the People's Republic of China. (II) Nurses worked in the pediatric outpatient department of Xiangyang No. 1 People's Hospital. (III) This study was approved by the Ethics Committee of Xiangyang No. 1 People's Hospital, and the nurses were informed of the purpose and process of the study and signed informed consent documents. The study was conducted in accordance with the Declaration of Helsinki (as revised in 2013).

\section{Exclusion criteria}

(I) Nurses did not work in the pediatric outpatient department. (II) Nurses could not complete humanistic care teaching model training. (III) Nurses had previously received the relevant training.

\section{Study methods}

In the control group, the nurses received a traditional pediatric nursing teaching model. Those who had worked in pediatric practice for more than 6 years were selected to teach clinical theoretical knowledge on pediatrics through courseware, conduct training on clinical practice skills, organize nurses to watch videos, perform demonstrations, and explain the operation essentials. In addition, the teachers also shouldered the responsibility to carry out related practice and provide explanations and demonstrations targeted at practice content with high error rates $(9,10)$.

In the experimental group, the nurses were subject to a traditional pediatric nursing teaching model combined with the humanistic care teaching model. Based on potential nursing risks in the pediatric outpatient department and the concept of clinical nursing care, rational teaching goals were set. Nurses had to fully understand the concept of nursing care before teaching commenced, then teachers comprehensively imparted the relevant knowledge of the humanistic care teaching model and delivered the essence of clinical nursing, which was also the process of showing care for children. In addition, the teachers established nursing care scenarios through digital multimedia and simulation during the teaching process. Teachers prepared stories and videos about nursing care and integrated them into teaching practice. During practice, they also wrote 
various clinical cases and organized role play to improve the ability of nurses to deal with verbal communication and handling emergency situations through simulation treatment drilling. The teaching evaluation system was divided into two parts, of which $25 \%$ was formal evaluation including daily attendance, activity participation, and classroom performance, and $75 \%$ as final evaluation. Additionally, the elements of the humanistic care teaching model were included in the system, and the scoring points were quantified and refined to highlight the importance of humanistic care and raise the attention of nurses.

\section{Observation indexes}

The clinical nursing ability of the nurses in both groups (primary outcome) after training was evaluated by adopting the Pediatric Nursing Staff Competence Scoring Scale made by our department, which included strain capacity, operational capacity, nurse-patient communication skills, autonomous learning ability, and teamwork ability, with each item scoring 10 points and higher scores indicating better working ability of nurses.

The professional identity of the nurses in both groups (primary outcome) was evaluated by adopting the Professional Identity Rating Scale For Health Nurses made by Xiangyang No. 1 People's Hospital, which was divided into five scoring items, with a total score of 10 points and each item scoring two points and higher scores indicating better professional identity of nurses.

The turnover intention of nurses in both groups (second outcome) was evaluated with reference to the Turnover Intention Scale (11), which included the motivation to find other jobs, the possibility of acquiring outside jobs, and the possibility of quitting the current job, with a total score of seven points for each item and higher scores indicating a stronger turnover intention of nurses.

In the face of different conflicts, the problem solving ability of nurses (primary outcome) was evaluated by the Pediatric Standardized Nursing Language Assessment Scale made by our department, and the causes of conflicts included children patients' factors, faults in work, charge factors, as well as unreasonable facilities and processes, with a total score of 30 points for each item. Higher scores indicated better ability to address nurse-patient conflicts.

Nursing satisfaction (primary outcome) was evaluated by the Nursing Satisfaction Questionnaire Of Children Patients proposed by the department, which included the items of waiting to see the doctor, health knowledge, ward environment, and nursing quality, with each item scoring a total of 15 points and higher scores indicating accompanying family members' higher satisfaction with the clinical nursing received.

\section{Statistical analysis}

All the study data were processed and statistically analyzed by SPSS21.0 software, and GraphPad Prism 7 (Graphpad Software, San Diego, USA) was used to draw the picture of the data. Measurement data were expressed by $(\bar{x} \pm s)$ and tested by $t$-test and enumeration data were expressed as [n (\%)] and tested by $\chi^{2}$ test. The differences had statistical significance when $\mathrm{P}<0.05$.

\section{Results}

Comparison of general information between the two groups

There were no significant differences in gender ratio, age, marital status, education, and employment status between the two groups $(\mathrm{P}>0.05)$, with comparability, as shown in Table 1 and Figure 1.

\section{Comparison of abilities after training between the two groups}

The strain capacity, operational capacity, nurse-patient communication skills, autonomous learning ability, and teamwork ability of the nurses in the experimental group after the training were significantly better than those in the control group $(\mathrm{P}<0.05)$, as shown in Table 2.

\section{Comparison of professional identity before and after training between the two groups}

The professional identity scores of nurses in both groups after training were significantly higher than those before training, and the scores in the experimental group after training were significantly higher than those in the control group $(\mathrm{P}<0.05)$, as shown in Figure 2.

\section{Comparison of turnover intention between the two groups}

The scores in the aspects associated with the motivation of nurses to find other jobs, the possibility of acquiring outside jobs, the possibility of quitting the current job, and the total scores of turnover intention in the experimental group were 
Table 1 Comparison of general information between the two groups

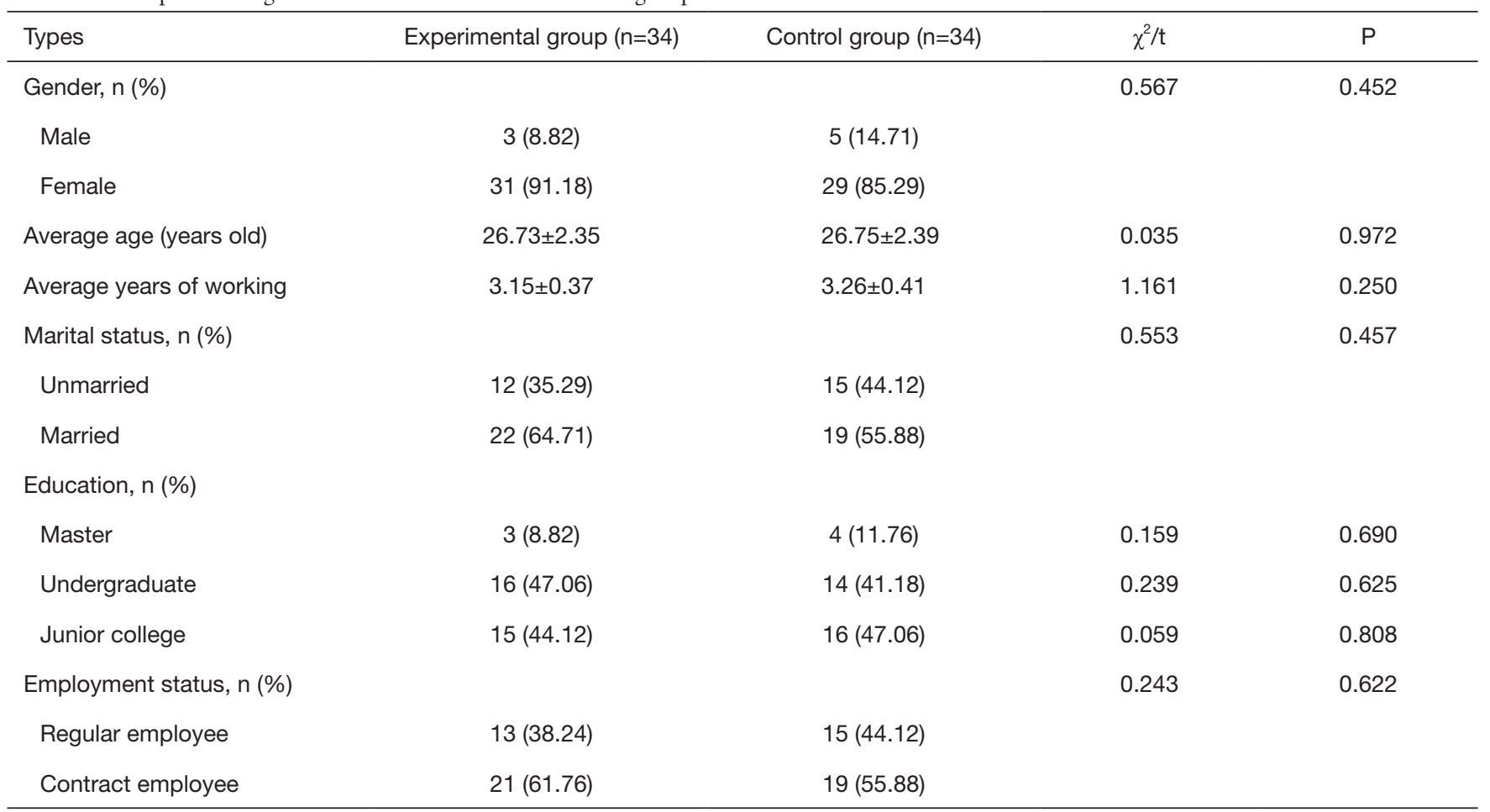

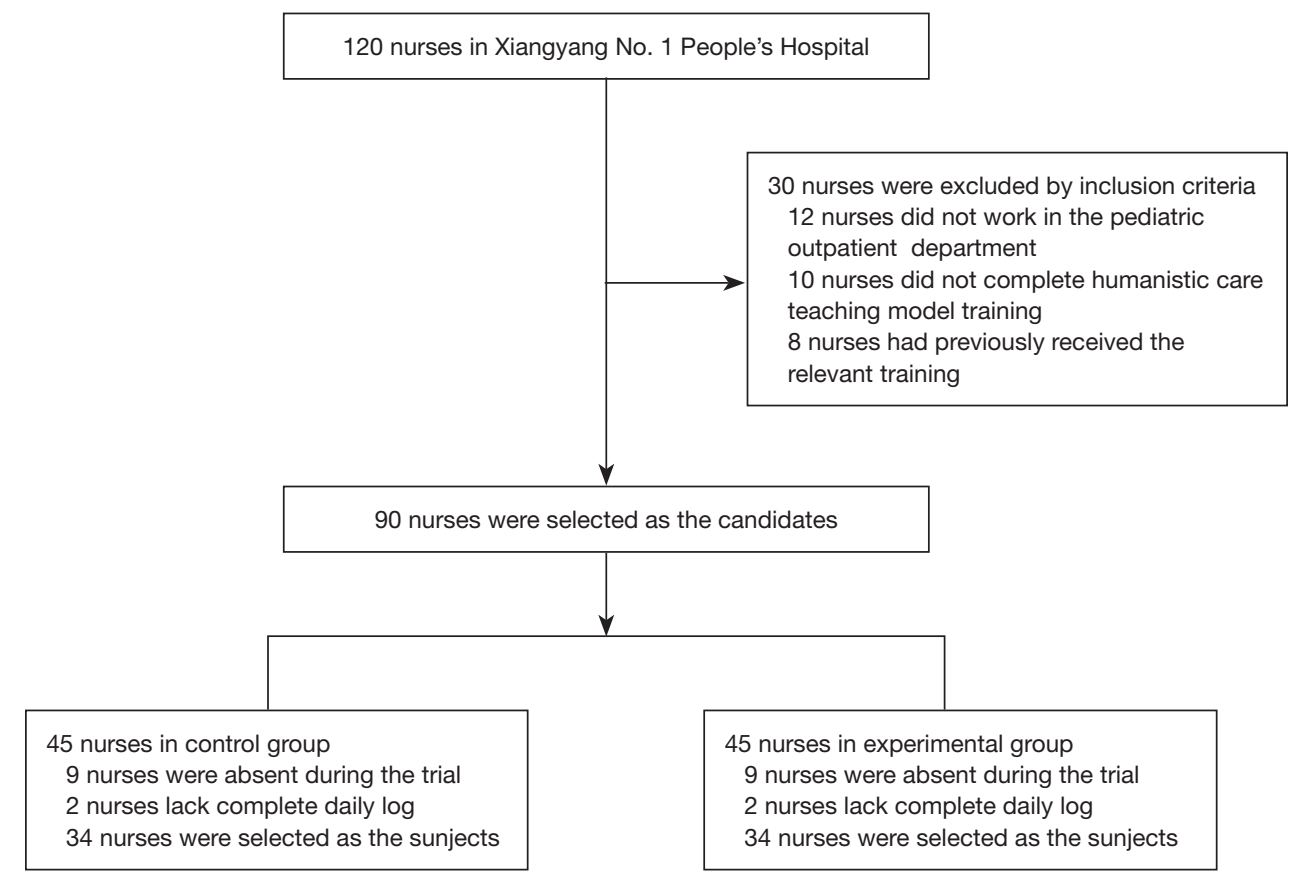

Figure 1 The flow diagram of subject selection. 
Table 2 Comparison of abilities after training between the two groups ( $x \pm \mathrm{s}$, points)

\begin{tabular}{lcccc}
\hline Abilities after training & Experimental group $(\mathrm{n}=34)$ & Control group $(\mathrm{n}=34)$ & $\mathrm{t}$ & $\mathrm{P}$ \\
\hline Strain capacity & $8.24 \pm 1.63$ & $7.41 \pm 1.57$ & 2.138 & 0.036 \\
Operational capacity & $8.48 \pm 1.26$ & $6.52 \pm 1.48$ & 5.88 & 0.000 \\
Nurse-patient communication skills & $8.63 \pm 1.15$ & $6.37 \pm 1.06$ & 8.426 & 0.000 \\
Autonomous learning ability & $8.19 \pm 0.59$ & $6.12 \pm 0.58$ & 14.589 & 0.000 \\
Teamwork ability & $8.38 \pm 0.42$ & $6.33 \pm 0.52$ & 17.883 & 0.000 \\
\hline
\end{tabular}

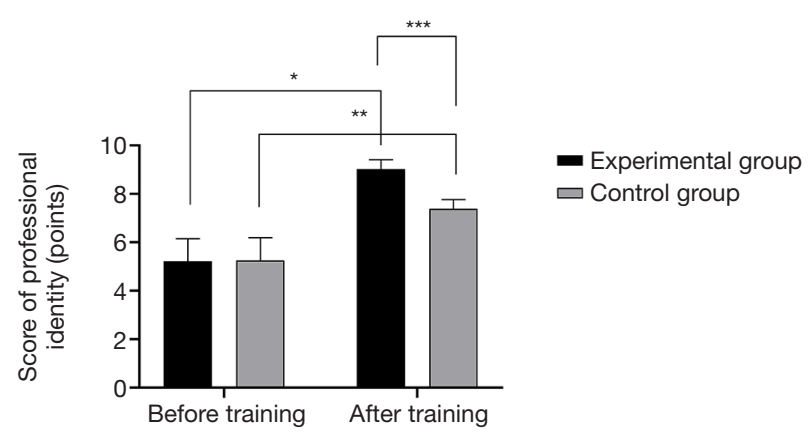

Figure 2 Comparison of professional identity before and after training between the two groups $(\bar{x} \pm \mathrm{s})$. The abscissa represented before and after training, while the ordinate represented the score of professional identity (points). The scores of professional identity in the experimental group before and after training were $4.56 \pm 1.32$ and $8.74 \pm 0.56$, respectively, and those in the control group were $4.58 \pm 1.34$ and $7.12 \pm 0.53$ points, respectively. * indicated that there were significant differences in the scores of professional identity in the experimental group before and after training $(\mathrm{t}=16.998$, $\mathrm{P}=0.000) .{ }^{* *}$ indicated that there were significant differences in the scores of professional identity in the control group before and after training $(\mathrm{t}=10.278, \mathrm{P}=0.000) .{ }^{* * *}$ indicated that there were significant differences in the scores of professional identity in the two groups after training $(\mathrm{t}=12.251, \mathrm{P}=0.000)$.

significantly lower than those in the control group $(\mathrm{P}<0.05)$, as shown in Table 3.

\section{Comparison of conflict solving ability after training between the two groups}

In the face of various conflicts, the problem solving ability of the nurses in the experimental group was significantly higher than that in the control group $(\mathrm{P}<0.05)$, as shown in Table 4.

\section{Comparison of clinical nursing satisfaction between the two groups}

The scores in the aspects of waiting to see the doctor, health knowledge, ward environment, and nursing quality in the experimental group were significantly higher than those in the control group $(\mathrm{P}<0.05)$, as shown in Table 5 .

\section{Discussion}

Interpersonal interactions are thwart with conflict, and in a hospital setting this exists among doctors, nurses, and patients and their families. This is particularly so in the pediatric outpatient department where much anxiety and stress will exacerbate nurse-patient conflicts and adversely affect clinical treatment (12-14). As nurses play a crucial role in linking doctors and patients' family members, they are the key players in addressing conflicts. However, because of the complexity of pediatric disease and dealing with patients and their families, a greater work pressure exists for pediatric nurses in comparison to other departments. When nurses face multiple work pressures and larger workloads, their ability to address conflicts reasonably is challenged, and improper handling of conflicts can reduce the sense of achievement felt by nursing staff, leading to higher turnover rates (15). The humanistic care teaching model is patientcentered and by focusing on the clinical practical demands of patients, can improve nursing comfort and accelerate disease rehabilitation. The model has been shown to be beneficial for pediatric nurses by promoting professional skills, improving nurse-patient communication ability, and avoiding nurse-patient conflicts (16-18).

Professional identity refers to the individual perception of career contents, goals, social values, and other factors. If this is consistent with the expectations and evaluation of the society, it means that the individual totally agrees with 
Table 3 Comparison of turnover intention between the two groups ( $x \pm \mathrm{s}$, points)

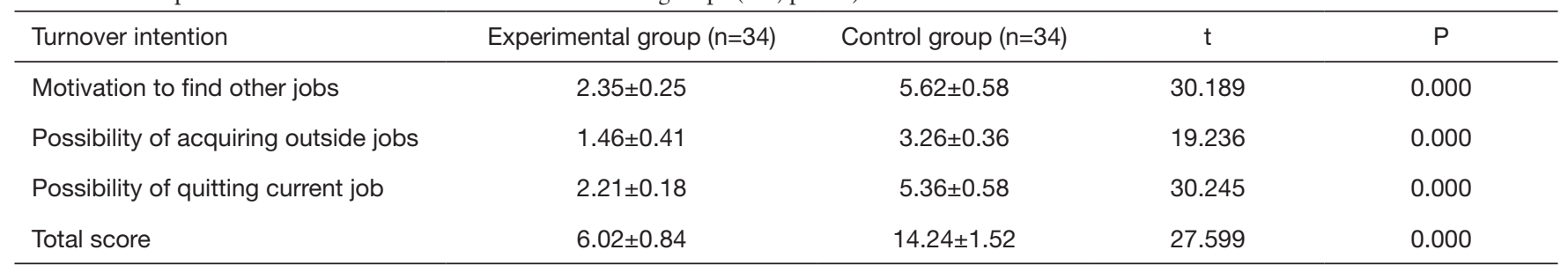

Table 4 Comparison of conflict solving ability after training between the two groups ( $x \pm \mathrm{s}$, points)

\begin{tabular}{lccc}
\hline Conflict solving ability & Experimental group $(\mathrm{n}=34)$ & Control group $(\mathrm{n}=34)$ & $\mathrm{t}$ \\
\hline Pediatric patient factors & $24.23 \pm 2.31$ & $17.42 \pm 2.19$ & 12.475 \\
Charge factors & $26.19 \pm 1.86$ & $16.53 \pm 1.53$ & 23.388 \\
Faults in work & $25.63 \pm 1.36$ & $17.34 \pm 1.74$ & 21.888 \\
Unreasonable facilities and processes & $24.37 \pm 2.32$ & $19.26 \pm 1.84$ & 0.000 \\
\hline
\end{tabular}

Table 5 Comparison of clinical nursing satisfaction between the two groups ( $x \pm$ s, points)

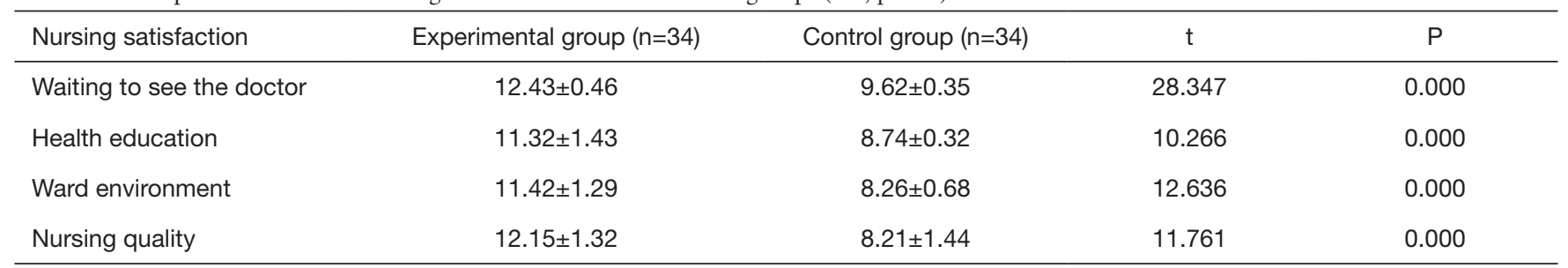

the career perception of other people or groups. Pancras et al. (19) have pointed out that professional identity is the basis for enhancing the professional quality of medical staff, and those with higher professional identity tend to have attained more job achievements. Our study found that the professional identity scores of nurses in the experimental group after training were significantly higher than those in the control group $(\mathrm{P}<0.05)$, suggesting that the humanistic care teaching model can improve the professional identity of pediatric nurses and reduce turnover rates. The caring ability of nurses is not innate and is gradually established based on an accumulation of experience (20-22). In this study, the humanistic care teaching model was established through setting teaching objectives and improving teaching methods, as well as teaching evaluation to guide pediatric nurses to improve nursing care ability and the quality of their care. In the teaching process, the most important thing is to create psychological resonance and emotional identity for nurses, and to improve their clinical problem solving ability by carrying out role-play and simulating real- life scenarios.

In this study, we confirmed that the ability of pediatric nurses to handle different nurse-patient conflicts after training in the experimental group was significantly better than that in the control group $(\mathrm{P}<0.05)$. Our results align with those of Wan et al. (23), who found that after nurses in a neurology department underwent the humanistic care teaching model, the scores of $12.38 \pm 2.31$ points of their conflict solving ability when dealing with patients were significantly higher than the $8.24 \pm 2.07$ points seen in a reference group. This shows the humanistic care teaching model can significantly improve the nursing-patient conflict solving ability of pediatric nurses, enhance their professional identity, and reduce turnover rates.

In conclusion, the humanistic care teaching model can effectively improve the professional identity of pediatric nurses, improve their problem solving ability to cope with various nurse-patient conflicts, and reduce turnover rates. This model is worthy of application and promotion in clinical nursing teaching. 


\section{Acknowledgments}

Funding: None.

\section{Footnote}

Reporting Checklist: The authors have completed the CONSORT reporting checklist. Available at https://dx.doi. org/10.21037/tp-21-214

Trial Protocol: Available at https://dx.doi.org/10.21037/tp21-214

Data Sharing Statement: Available at https://dx.doi. org/10.21037/tp-21-214

Conflicts of Interest: All authors have completed the ICMJE uniform disclosure form (available at https://dx.doi. org/10.21037/tp-21-214). The authors have no conflicts of interest to declare.

Ethical Statement: The authors are accountable for all aspects of the work in ensuring that questions related to the accuracy or integrity of any part of the work are appropriately investigated and resolved. This study was approved by the Ethics Committee of Xiangyang No. 1 People's Hospital, and the nurses were informed of the purpose and process of the study and signed informed consent documents. The study was conducted in accordance with the Declaration of Helsinki (as revised in 2013).

Open Access Statement: This is an Open Access article distributed in accordance with the Creative Commons Attribution-NonCommercial-NoDerivs 4.0 International License (CC BY-NC-ND 4.0), which permits the noncommercial replication and distribution of the article with the strict proviso that no changes or edits are made and the original work is properly cited (including links to both the formal publication through the relevant DOI and the license). See: https://creativecommons.org/licenses/by-nc-nd/4.0/.

\section{References}

1. Kartha MR, McCrone P. Cost-effectiveness of staffing levels on conflict and containment on psychiatric wards in England. J Psychiatr Ment Health Nurs 2019;26:337-46.

2. Gergerich E, Boland D, Scott MA. Hierarchies in interprofessional training. J Interprof Care 2019;33:528-35.
3. Alahmad G, Al-Kamli H, Alzahrani H. Ethical Challenges of Pediatric Cancer Care: Interviews With Nurses in Saudi Arabia. Cancer Control 2020;27:1073274820917210.

4. Johnstone MJ, Turale S. Nurses' experiences of ethical preparedness for public health emergencies and healthcare disasters: a systematic review of qualitative evidence. Nurs Health Sci 2014;16:67-77.

5. Hernon O, Dalton R, Dowling M. Clinical research nurses' expectations and realities of their role: A qualitative evidence synthesis. J Clin Nurs 2020;29:667-83.

6. Warembourg M, Lonca N, Filleron A, et al. Assessment of anti-infective medication adherence in pediatric outpatients. Eur J Pediatr 2020;179:1343-51.

7. McEvoy H, Borsook D, Holmes SA. Clinical features and sex differences in pediatric post-traumatic headache: A retrospective chart review at a Boston area concussion clinic. Cephalalgia 2020;40:701-11.

8. Bauer JM, Welling SE, Ross KE, et al. What Should I Wear to Clinic? A National Survey of Pediatric Orthopaedic Patients and Parents. J Pediatr Orthop 2020;40:e1010-6.

9. Kleinendorst L, Abawi O, van der Voorn B, et al. Identifying underlying medical causes of pediatric obesity: Results of a systematic diagnostic approach in a pediatric obesity center. PLoS One 2020;15:e0232990.

10. Boppana SS, Miller R, Wrona A, et al. Barriers to Outpatient Pediatric Chronic Pain Clinic Participation Among Referred Patients. Clin Pediatr (Phila) 2020;59:859-64.

11. Fenton J, Posa M, Kelly M, et al. Impact of a Point-of-care Respiratory PCR Panel in a Pediatric Clinic on Postvisit Communication and Follow-up Visits. Pediatr Infect Dis J 2020;39:e282-3.

12. Benjamin JC, Groner J, Walton J, et al. A Blended Curriculum to Improve Resident Physical Exam Skills for Patients With Neuromuscular Disability. MedEdPORTAL 2019;15:10792.

13. Atkins AI, Cole JG. A 5-Year-Old Child Who Presents to the General Pediatric Clinic for a Well-Child Check Is Found to Be in Hypoxic Respiratory Distress. Clin Pediatr (Phila) 2020;59:1123-5.

14. Tsai $\mathrm{Y}, \mathrm{Wu} \mathrm{SW}$. The relationships between organisational citizenship behaviour, job satisfaction and turnover intention. J Clin Nurs 2010;19:3564-74.

15. Chang YP, Lee DC, Wang HH. Violence-prevention climate in the turnover intention of nurses experiencing workplace violence and work frustration. J Nurs Manag 2018;26:961-71. 
16. Onyishi IE, Enwereuzor IK, Ogbonna MN, et al. Role of Career Satisfaction in Basic Psychological Needs Satisfaction and Career Commitment of Nurses in Nigeria: A Self-Determination Theory Perspective. J Nurs Scholarsh 2019;51:470-9.

17. Zhang W, Meng H, Yang S, et al. The Influence of Professional Identity, Job Satisfaction, and Work Engagement on Turnover Intention among Township Health Inspectors in China. Int J Environ Res Public Health 2018;15:988.

18. Smith TD. An assessment of safety climate, job satisfaction and turnover intention relationships using a national sample of workers from the USA. Int J Occup Saf Ergon 2018;24:27-34.

19. Pancras G, Shayo J, Anaeli A. Non-medical facilitators and barriers towards accessing haemodialysis services: an exploration of ethical challenges. BMC Nephrol 2018;19:342.

Cite this article as: Zhong $\mathrm{X}$, Liu X, Sheng Y. The effect of the humanistic care teaching model on nurse patient conflict and nurse turnover intention in a pediatric outpatient department: results of a randomized trial. Transl Pediatr 2021;10(8):2016-2023. doi: 10.21037/tp-21-214
20. Duan X, Ni X, Shi L, et al. The impact of workplace violence on job satisfaction, job burnout, and turnover intention: the mediating role of social support. Health Qual Life Outcomes 2019;17:93.

21. Lee E, Jang I. Nurses' Fatigue, Job Stress, Organizational Culture, and Turnover Intention: A Culture-Work-Health Model. West J Nurs Res 2020;42:108-16.

22. De Simone S, Planta A, Cicotto G. The role of job satisfaction, work engagement, self-efficacy and agentic capacities on nurses' turnover intention and patient satisfaction. Appl Nurs Res 2018;39:130-40.

23. Wan Q, Li Z, Zhou W, et al. Effects of work environment and job characteristics on the turnover intention of experienced nurses: The mediating role of work engagement. J Adv Nurs 2018;74:1332-41.

(English Language Editor: B. Draper) 